Preprint typeset in JHEP style - HYPER VERSION

\title{
Linear broadening of the confining string in Yang-Mills theory at low temperature
}

\author{
F. Gliozzi \\ Dipartimento di Fisica Teorica, Università di Torino, and \\ INFN, Sezione di Torino, via P. Giuria 1, 10125 Torino, Italy. \\ E-mail: gliozzi@to.infn.it
}

\author{
M. Pepe \\ INFN, Sezione di Milano-Bicocca \\ Edificio U2, Piazza della Scienza 3, 20126 Milano, Italy. \\ E-mail: pepe@mib.infn.it
}

\section{U.-J. Wiese \\ Albert Einstein Center for Fundamental Physics \\ Institute for Theoretical Physics, Bern University \\ Sidlerstr. 5, 3012 Bern, Switzerland. \\ E-mail: wiese@itp.unibe.ch}

\begin{abstract}
The logarithmic broadening predicted by the systematic low-energy effective field theory for the confining string has recently been verified in numerical simulations of $(2+1)$-d $S U(2)$ lattice Yang-Mills theory at zero temperature. The same effective theory predicts linear broadening of the string at low non-zero temperature. In this paper, we verify this prediction by comparison with very precise Monte Carlo data. The comparison involves no additional adjustable parameters, because the low-energy constants of the effective theory have already been fixed at zero temperature. It yields very good agreement between the underlying Yang-Mills theory and the effective string theory.
\end{abstract}

KeYwords: Nonperturbative Effects, Lattice Gauge Field Theories, Lattice Quantum Field Theory, Bosonic Strings. 


\section{Contents}

1. Introduction 1

2. Analytic Results of the Low-Energy Effective String Theory 2

3. Numerical Results for $(2+1)$-d $S U(2)$ Lattice Yang-Mills Theory

4. Conclusions 7

\section{Introduction}

At low temperature, in Yang-Mills theory static quarks and anti-quarks are confined to one another by an unbreakable string. The string itself is an interesting object with peculiar dynamical properties. The relevant energy scale for these dynamics is set by the string tension $\sigma$, which defines the asymptotic slope of the linearly rising quark-anti-quark potential. The string tension also controls the amplitude of transverse fluctuations of the string. As was pointed out a long time ago by Lüscher, Symanzik, and Weisz [1], these fluctuations are described by a systematic low-energy effective string theory. In fact, the transverse fluctuations are massless modes (i.e. Goldstone excitations) of a spontaneously broken translational symmetry of the string world-sheet, also known as capillary waves in condensed matter physics. The effective string theory plays the role of chiral perturbation theory for these Goldstone modes. Using the effective theory, Lüscher has calculated the leading correction to the linear quark-anti-quark potential

$$
V(r)=\sigma r-\frac{\pi(d-2)}{24 r}+\mathcal{O}\left(1 / r^{3}\right),
$$

where $d$ is the space-time dimension and $(d-2)$ counts the directions transverse to the string world-sheet $[1,2]$. Accurate numerical simulations have shown the validity of that expectation [3-12]. The effective theory also accounts for the string width [13]. Due to its transverse fluctuations, the string broadens as the quark sources are separated. The cross-sectional area swept out by the fluctuating string increases logarithmically with the distance $r$ between the static sources, i.e.

$$
w_{l o}^{2}(r / 2)=\frac{d-2}{2 \pi \sigma} \log \left(r / r_{0}\right) .
$$

Here $w_{l o}(r / 2)$ is the leading-order string width at the distance $r / 2$, half-way between the external static quark sources, and $r_{0}$ is a length scale that enters the effective string theory as a low-energy parameter. The above expression for the width is universal and applies to strings in Yang-Mills theory as well as to fluctuating interfaces between different phases of 
condensed matter. It has been verified in numerical simulations for the interfaces separating the two low-temperature phases of the 3-d Ising model above the roughening transition [14]. Similar results have been obtained in a $(2+1)-d \mathbb{Z}(4)$ gauge theory [15]. Since numerical lattice calculations of the string width in non-Abelian gauge theories are computationally very challenging, early calculations were affected by large statistical errors and did thus not yield conclusive results [16]. Only recently, using the very efficient Lüscher-Weisz multi-level algorithm $[6,17]$, the logarithmically divergent string width has been accurately verified by simulating $(2+1)$-d $S U(2)$ lattice Yang-Mills theory [18]. Recent results on the effective string description of the color flux tube in $(2+1)$-d $S U(N)$ Yang-Mills theory can be found in $[19,20]$.

At low temperature $T=1 / \beta$, the extent $\beta$ of the periodic Euclidean time direction enters as another length scale in the low-energy effective string theory. At low $T \ll \sqrt{\sigma}$, the temperature effects on the string tension and the string broadening are again accessible to a systematic treatment. At leading order, the temperature-dependent string tension is given by

$$
\sigma(\beta)=\sigma-\frac{\pi(d-2)}{6 \beta^{2}} .
$$

Similarly, the leading-order string width at a distance $r / 2 \gg \beta$ takes the form [21, 22]

$$
w_{l o}^{2}(r / 2)=\frac{d-2}{2 \pi \sigma} \log \frac{\beta}{4 r_{0}}+\frac{d-2}{4 \beta \sigma} r+\mathcal{O}\left(\mathrm{e}^{-2 \pi r / \beta}\right) .
$$

Hence, at non-zero temperature the confining string broadens linearly with the distance between the external static quarks. Numerical evidence for linear string broadening at finite temperature was presented for fluctuating interfaces in the 3-d Ising model in $[21,22]$ and, recently, for $(3+1)$-d $S U(3)$ Yang-Mills theory in [23]. In this paper, using the LüscherWeisz multi-level simulation technique, we perform very accurate Monte Carlo calculations of the temperature-dependent string width in $(2+1)$-d $S U(2)$ Yang-Mills theory. The numerical results are then compared with the corresponding analytic predictions of the systematic low-energy effective string theory at the 2-loop level [24]. Since the low-energy parameters of the effective theory were previously fixed by a comparison with Monte Carlo data at zero temperature, there are no adjustable parameters. We find perfect agreement between the numerical data and the analytic predictions, which lends further support to the quantitative validity of the effective theory.

The rest of this paper is organized as follows. Section 2 describes the analytic calculations in the low-energy effective string theory, which are then compared with the numerical results obtained in section 3 . Section 4 contains our conclusions.

\section{Analytic Results of the Low-Energy Effective String Theory}

Let us consider the systematic low-energy effective theory describing the transverse fluctuations of the string world sheet in $(2+1)$-d Yang-Mills theory. In terms of the height variable $h(x, t)$ the corresponding Euclidean action is given by

$$
S[h]=\int_{0}^{\beta} d t \int_{0}^{r} d x \frac{\sigma}{2}\left[\partial_{\mu} h \partial_{\mu} h-\frac{1}{4}\left(\partial_{\mu} h \partial_{\mu} h\right)^{2}\right] .
$$


Here $x \in[0, r]$ and $t \in[0, \beta]$ parameterize the 2-d base-space. Since the string ends at static quark charges separated by a distance $r$, its fluctuation field obeys the boundary condition $h(0, t)=h(r, t)=0$. The boundary conditions explicitly break translation invariance and one might have expected boundary terms to be present in the effective action as well [6]. Remarkably, due to open-closed string duality, such terms are absent and the prefactor of the first sub-leading term is uniquely determined [25]. This behavior even extends to the next order six-derivative term. Indeed, up to that order the effective action coincides with the one of the Nambu-Goto string [26-28].

The confining string of a lattice Yang-Mills theory suffers from lattice artifacts which disappear only in the continuum limit. Away from the continuum limit, in the strong coupling regime, the lattice string is rigid, i.e. it follows the discrete lattice steps and does not even support massless fluctuations. When one crosses the roughening transition at intermediate values of the coupling, one enters the rough phase with massless Goldstone excitations of the string, to which the low-energy effective string theory applies. Since the lattice theory is invariant only under discrete rotations and not under the full Poincaré group, before one reaches the continuum limit two additional terms proportional to $\sum_{\mu=1,2} \partial_{\mu} \partial_{\mu} h \partial_{\mu} \partial_{\mu} h$ and to $\sum_{\mu=1,2}\left(\partial_{\mu} h\right)^{4}$ enter the effective theory in the bulk. Since these terms contain four derivatives, they are of sub-leading order. Hence, they have no effect on the Lüscher term or on the leading behavior of the string width. As a result, the Lüscher term is indeed completely universal. Provided its world-sheet is rough, even a lattice string supports exactly massless modes which contribute $-\pi / 24 r$ to the static quark potential. In particular, this term is not affected by lattice spacing artifacts, since such effects are of sub-leading order. In the following, we will work sufficiently close to the continuum limit of the lattice theory. In that case, the effective action of eq.(2.1) without the additional non-rotational-invariant terms is sufficient.

Recently, an analytic expression for the string width at zero temperature has been derived from the effective string theory at the 2-loop level [24]

$$
w^{2}(r / 2)=\left(1+\frac{4 \pi f(\tau)}{\sigma r^{2}}\right) w_{l o}^{2}(r / 2)-\frac{f(\tau)+g(\tau)}{\sigma^{2} r^{2}}
$$

where

$$
f(\tau)=\frac{E_{2}(\tau)-4 E_{2}(2 \tau)}{48}, \quad g(\tau)=i \pi \tau\left(\frac{E_{2}(\tau)}{12}-q \frac{d}{d q}\right)\left(f(\tau)+\frac{E_{2}(\tau)}{16}\right)+\frac{E_{2}(\tau)}{96} .
$$

The parameter $q=\mathrm{e}^{2 \pi i \tau}$ depends on the ratio $\tau=i \beta /(2 r)$ and

$$
E_{2}(\tau)=1-24 \sum_{n=1}^{\infty} \frac{n q^{n}}{1-q^{n}},
$$

is the first Eisenstein series. The leading-order expression for the width

$$
w_{l o}^{2}(r / 2)=\frac{1}{2 \pi \sigma} \log \left(\frac{r}{r_{0}}\right)+\frac{1}{\pi \sigma} \log \left(\frac{\eta(2 \tau)}{\eta(\tau)^{2}}\right)
$$


contains the Dedekind $\eta$-function

$$
\eta(\tau)=q^{\frac{1}{24}} \prod_{n=1}^{\infty}\left(1-q^{n}\right)
$$

This function and the Eisenstein series $E_{2}$ obey the following transformations under the inversion $\tau \rightarrow-\tau^{-1}$

$$
\eta(\tau)=\frac{1}{\sqrt{-i \tau}} \eta\left(-\frac{1}{\tau}\right), \quad E_{2}(\tau)=\frac{1}{\tau^{2}} E_{2}\left(-\frac{1}{\tau}\right)-\frac{6}{i \pi \tau} .
$$

Using these relations, the expression of eq.(2.2) in the zero-temperature limit, $\beta \gg r$, is readily converted into an expression in the limit $r \gg \beta$. In this case, the temperature is still small, but the string length $r$ is much larger than the inverse temperature $\beta$.

For completeness, we present also the expression for the force $F$ at finite temperature, defined as the first derivative of the potential. At leading order the force is given by

$$
F_{l o}(r)=\sigma+\frac{1}{2 \beta r}-\frac{\pi}{6 \beta^{2}} E_{2}\left(-\frac{1}{\tau}\right) .
$$

The potential at next-to-leading order has been obtained from the computation of the partition function using the effective string action of eq.22.1) [29]. Then the force is given by the expression

$$
F(r)=F_{l o}(r)-\frac{\pi^{2}}{72 \sigma \beta^{4}} \frac{d}{d r}\left[r\left(2 E_{4}\left(-\frac{1}{\tau}\right)-E_{2}^{2}\left(-\frac{1}{\tau}\right)\right)-\frac{9 \beta^{2}}{\pi^{2} r}+\frac{6 \beta}{\pi} E_{2}\left(-\frac{1}{\tau}\right)\right]
$$

This equation shows that the 2-loop string tension at finite temperature is given by

$$
\sigma(\beta)=\sigma-\frac{\pi}{6 \beta^{2}}-\frac{\pi^{2}}{72 \sigma \beta^{4}}
$$

\section{Numerical Results for $(2+1)$-d $S U(2)$ Lattice Yang-Mills Theory}

Since it is least problematical in numerical simulations, we concentrate on $(2+1)$-d $S U(2)$ Yang-Mills theory on a cubic lattice of size $L \times L \times \beta$, with the Euclidean time extent $\beta$ determining the inverse temperature. The partition function is then given by

$$
Z=\int \mathcal{D} U \exp (-S[U])=\prod_{x, \mu} \int_{S U(2)} d U_{x, \mu} \exp (-S[U]) .
$$

Here $d U_{x, \mu}$ denotes the local gauge invariant Haar measure for a parallel transporter variable $U_{x, \mu} \in S U(2)$ located on the link $(x, \mu)$. We use the standard Wilson plaquette action

$$
S[U]=-\frac{2}{g^{2}} \sum_{x, \mu, \nu} \operatorname{Tr}\left[U_{x, \mu} U_{x+\hat{\mu}, \nu} U_{x+\hat{\nu}, \mu}^{\dagger} U_{x, \nu}^{\dagger}\right],
$$

where $g$ is the bare gauge coupling and $\hat{\mu}$ is a unit-vector pointing in the $\mu$-direction. As usual in lattice calculations, all physical quantities are expressed in units of the lattice spacing which is set to 1. 
The external static quarks at the two ends of the confining string are represented by Polyakov loops

$$
\Phi_{x}=\operatorname{Tr}\left[\prod_{t=1}^{\beta} U_{x+t \hat{2}, 2}\right],
$$

which wind around the periodic Euclidean time direction. We choose the 2-direction to represent Euclidean time. When two external quarks are separated in the 1-direction, the world-sheet of the confining string extends in the space-time directions $\mu=1,2$, while the string fluctuates in the transverse 3-direction. The static quark potential $V(r)$ is extracted from the Polyakov loop correlation function

$$
\left\langle\Phi_{0} \Phi_{r}\right\rangle=\frac{1}{Z} \int \mathcal{D} U \Phi_{0} \Phi_{r} \exp (-S[U]) \sim \exp (-\beta V(r)),
$$

in the zero-temperature limit $\beta \rightarrow \infty$.

The force between the two static quarks is extracted from the discrete derivative of the static potential

$$
F(\bar{r})=V(r+1)-V(r)=-\frac{1}{\beta} \log \frac{\left\langle\Phi_{0} \Phi_{r+1}\right\rangle}{\left\langle\Phi_{0} \Phi_{r}\right\rangle},
$$

where, writing explicitly the lattice spacing $a, \bar{r}=r+\frac{a}{2}+\mathcal{O}\left(a^{2}\right)$ is an improved definition of the point where the force is computed [30].

The width of the string is determined from the connected correlation function

$$
C\left(x_{3}\right)=\frac{\left\langle\Phi_{0} \Phi_{r} P_{x}\right\rangle}{\left\langle\Phi_{0} \Phi_{r}\right\rangle}-\left\langle P_{x}\right\rangle
$$

of a pair of Polyakov loops with a single plaquette

$$
P_{x}=\operatorname{Tr}\left[U_{x, 1} U_{x+\hat{1}, 2} U_{x+\hat{2}, 1}^{\dagger} U_{x, 2}^{\dagger}\right]
$$

in the 1-2 plane, which measures the color-electric field along the 1-direction of the string as a function of the transverse displacement $x_{3}$. The plaquette is located at the site $x=\left(r / 2, t, x_{3}\right)$ and thus measures the transverse fluctuations of the color flux tube at the maximal distance $r / 2$ from the external quark charges.

We have performed numerical simulations at the bare gauge coupling $4 / g^{2}=9.0$ on a $90^{2} \times 16$ lattice, corresponding to $T / T_{c} \approx 0.38$. We have used the Lüscher-Weisz technique $[6,17]$ with a single level and with slices of thickness 4 . In table 1 we report the number of sub-lattice updates for various ranges of distances between the static sources.

In figure 1, we plot the force between the two static quarks as a function of the distance between the sources. The dotted line is the force in the leading order approximation of eq.(2.8). The solid line is the next-to-leading order approximation given by eq.(2.9). It is important to note that there is no free parameter and $\sigma=0.025897(15)$ is the zero-temperature string tension. The next-to-leading order expression already provides a very good description of the numerical data. Considering the accuracy of the data, it is reasonable to expect that the residual tiny discrepancy can be described by the next-tonext-to-leading order correction. 


\begin{tabular}{|c|c|}
\hline$r$ range & updates \\
\hline $4-8$ & 3200 \\
$9-15$ & 24000 \\
$16-22$ & 32000 \\
$23-25$ & 80000 \\
\hline
\end{tabular}

Table 1: Number of sub-lattice updates of the single level Lüscher-Weisz algorithm for various ranges of distances between the static sources.

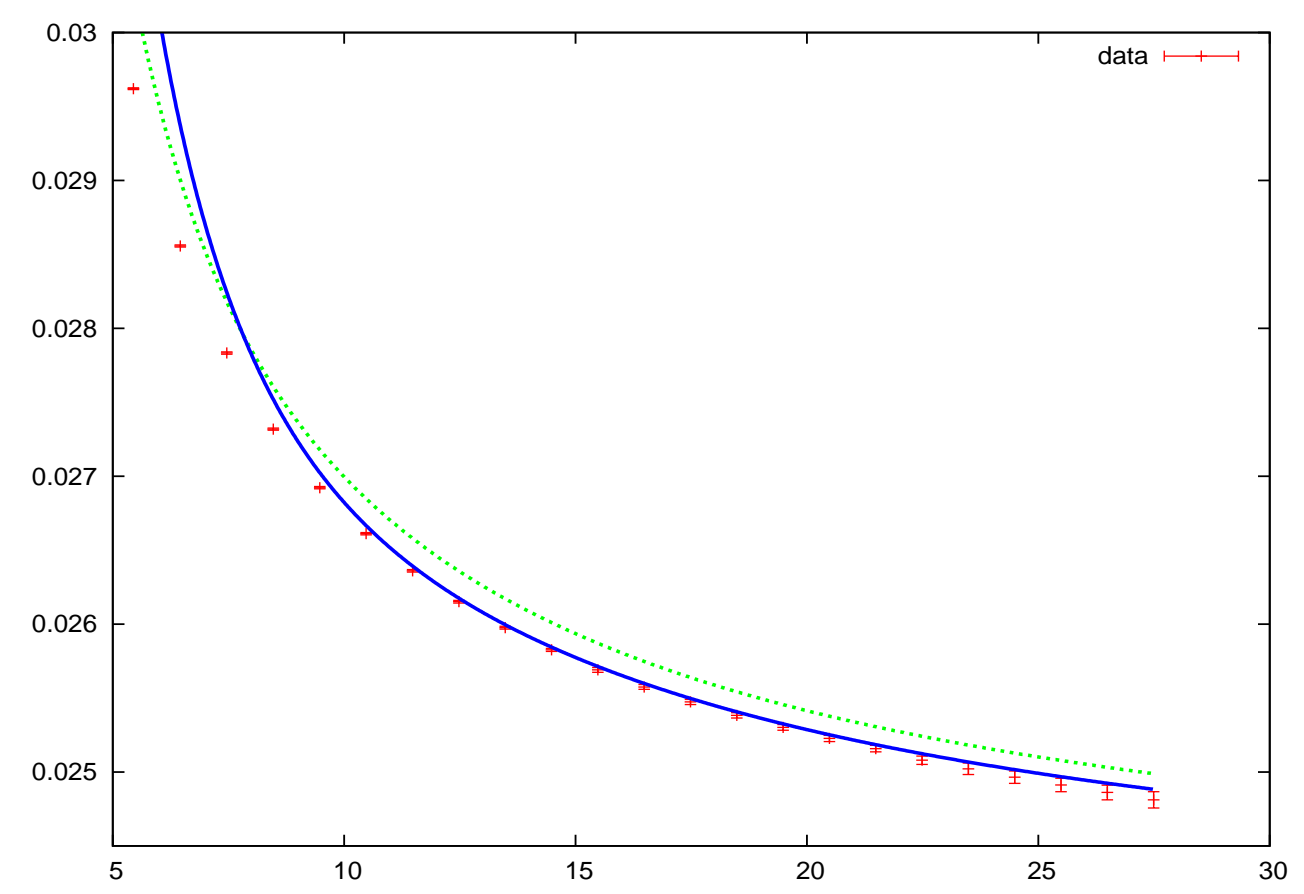

Figure 1: The force $F(\bar{r})$ as a function of the distance between the two static quarks. The dotted line and the solid line are the leading order approximation of eq.(2.8) and the next-to-leading order approximation of eq. 2.9 ), respectively.

For the measurement of the string width, the correlation function is fit to the ansatz

$$
\frac{\left\langle\Phi_{0} \Phi_{r} P_{x}\right\rangle}{\left\langle\Phi_{0} \Phi_{r}\right\rangle}=A \exp \left(-x_{3}^{2} / s\right) \frac{1+B \exp \left(-x_{3}^{2} / s\right)}{1+D \exp \left(-x_{3}^{2} / s\right)}+K,
$$

and the squared width of the string is then obtained as the second moment of the correlation function

$$
w^{2}(r / 2)=\frac{\int d x_{3} x_{3}^{2} C\left(x_{3}\right)}{\int d x_{3} C\left(x_{3}\right)} .
$$

In figure 2, we show the bell-shaped correlation function $\left\langle\Phi_{0} \Phi_{r} P_{x}\right\rangle /\left\langle\Phi_{0} \Phi_{r}\right\rangle$ as a function of the transverse displacement of the plaquette from the plane of the string world-sheet. The data refer to a pair of static sources at fixed distance $r=17$. The solid line is a fit of the data using the ansatz (3.8).

The scale that determines the logarithmic broadening of the string at zero temperature was previously determined as $r_{0}=2.26(2)$ such that $r_{0} \sqrt{\sigma}=0.364(3)$ [18]. It is important 


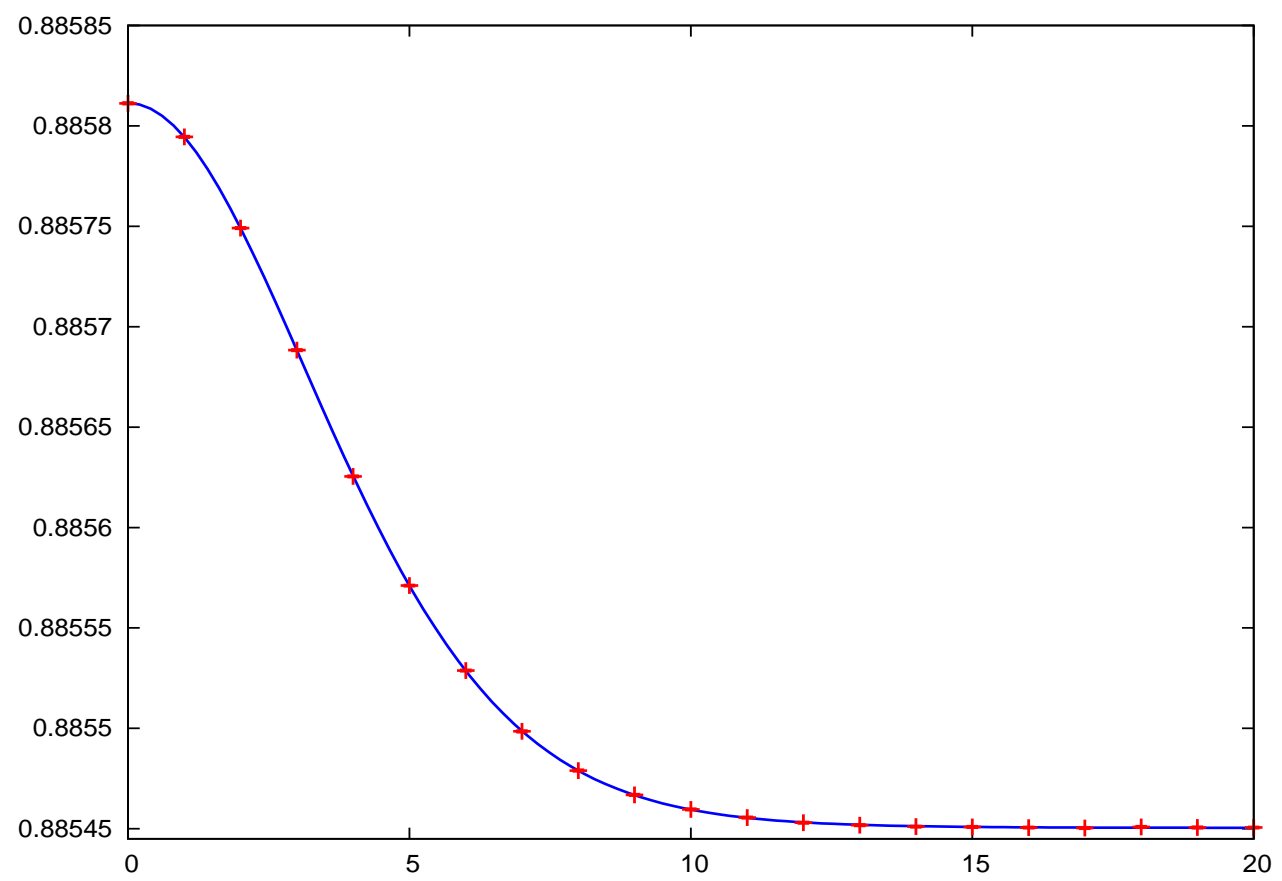

Figure 2: The ratio $\frac{\left\langle\Phi_{0} \Phi_{r} P_{x}\right\rangle}{\left\langle\Phi_{0} \Phi_{r}\right\rangle}$ as a function of the transverse displacement $x_{3}$ of the plaquette from the plane of the string world-sheet. The distance between the two static sources is $r=17$. The solid curve is a fit using eq.(3.8).

to note that $\sigma$ and $r_{0}$ are the only low-energy parameters entering the 2-loop formula for the width of the flux tube. Hence the theoretical prediction resulting from the low-energy effective theory is completely determined without any additional adjustable parameter.

In figure 3 we report the observed dependence of the flux tube width on the distance between the static sources as resulting from numerical Monte Carlo simulations. The two solid lines represent the fully constrained prediction of the low-energy effective string theory corresponding to the choices $r_{0}=2.26 \pm 0.02$. The numerical data show the expected linear behavior for sufficiently large separations of the sources and the agreement with the analytic formula turns out to be excellent.

Finally, it is important to notice that the next-to-leading expression (2.2) for the width is not sensitive to the next-to-leading correction to the string tension. In fact, the 2-loop result for the width goes up to corrections of order $1 /\left(\sigma \beta^{2}\right)$ and so the corrections of order $1 /\left(\sigma \beta^{2}\right)^{2}$ to the string tension will appear as 3-loop corrections to the width. Looking at the data for the width in figure 3 , the accuracy is such that we are not very sensitive to the next-to-leading correction to the string tension and one cannot detect the tiny difference that the direct measurement of the force in figure 11 shows.

\section{Conclusions}

Using the Lüscher-Weisz multi-level simulation technique, we have performed high-accuracy numerical simulations of the confining string connecting two static quarks in $(2+1)$-d $S U(2)$ 


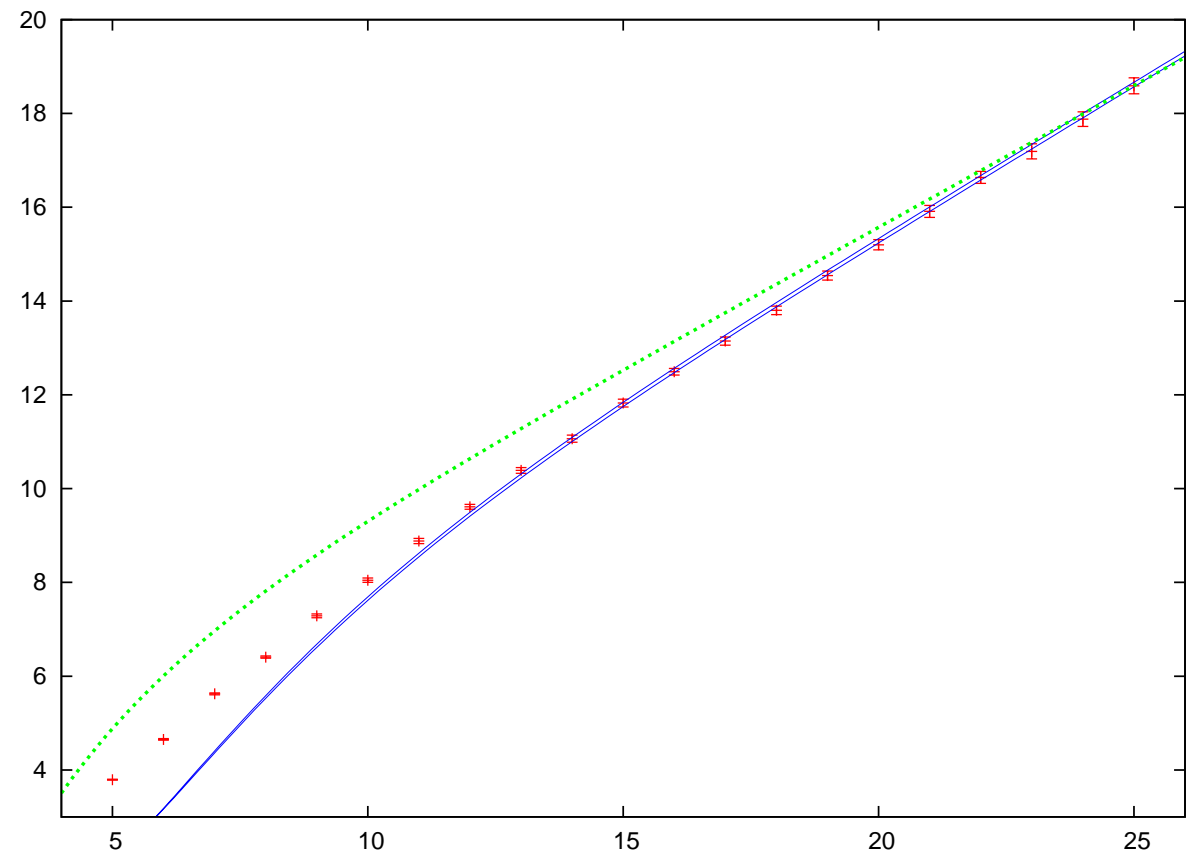

Figure 3: The squared width of the confining string at its midpoint $w^{2}(r / 2)$ as a function of the separation of the static sources $r$. The dotted line is the leading order prediction of the low-energy effective string theory using $r_{0}=2.26$. The two solid lines represent the 2-loop prediction using $r_{0}=2.26 \pm 0.02$.

Yang-Mills theory. The results for the finite temperature string width have been compared with analytic 2-loop calculations performed in the corresponding systematic low-energy effective string theory. Since the low-energy parameters $\sigma$ and $r_{0}$ were already determined at zero temperature, there were no further adjustable parameters in the comparison of the effective theory with the numerical simulations of the lattice Yang-Mills theory. Remarkably, there is perfect agreement between the underlying Yang-Mills theory and the effective string theory, thus confirming the quantitative correctness of the latter. With sufficient numerical resources (which are still modest on today's standards in lattice field theory) it is certainly feasible to extend our study to other gauge groups and to $(3+1)$ dimensions. A similar investigation in $(3+1)$-d $S U(3)$ Yang-Mills theory would definitely be possible. In particular, it would be interesting to investigate whether the effective theory works equally well in the numerically accessible regime as in the $(2+1)$-d case investigated here.

\section{Acknowledgements}

We gratefully acknowledge helpful discussions with M. Caselle and F. Niedermayer. This work is supported in part by funds provided by the Schweizerischer Nationalfonds (SNF). The "Albert Einstein Center for Fundamental Physics" at Bern University is supported by the "Innovations- und Kooperationsprojekt C-13" of the Schweizerische Universitätskonferenz (SUK/CRUS). 


\section{References}

[1] M. Lüscher, K. Symanzik, and P. Weisz, Nucl. Phys. B173 (1980) 365.

[2] M. Lüscher, Nucl. Phys. B180 (1981) 317.

[3] M. Hasenbusch and K. Pinn, Physica A192 (1993) 342.

[4] M. Caselle, R. Fiore, F. Gliozzi, M. Hasenbusch, and P. Provero, Nucl. Phys. B486 (1997) 245.

[5] K. J. Juge, J. Kuti, and C. Morningstar, Phys. Rev. Lett. 90 (2003) 161601.

[6] M. Lüscher and P. Weisz, JHEP 0207 (2002) 049.

[7] M. Caselle, M. Pepe, and A. Rago, JHEP 0410 (2004) 005.

[8] M. Caselle, M. Hasenbusch, and M. Panero, JHEP 0603 (2006) 084.

[9] N. D. Hari Dass and P. Majumdar, JHEP 0610 (2006) 020.

[10] B. Bringoltz and M. Teper, Phys. Lett. B663 (2008) 429.

[11] A. Athenodorou, B. Bringoltz, and M. Teper, JHEP 0905 (2009) 019.

[12] B. B. Brandt and P. Majumdar, Phys. Lett. B682 (2009) 253.

[13] M. Lüscher, G. Münster, and P. Weisz, Nucl. Phys. B180 (1981) 1.

[14] M. Caselle, F. Gliozzi, U. Magnea, and S. Vinti, Nucl. Phys. B460 (1996) 397.

[15] P. Giudice, F. Gliozzi, and S. Lottini, JHEP 0701 (2007) 084.

[16] G. Bali, K. Schilling, and C. Schlichter, Phys. Rev. D51 (1995) 5165.

[17] M. Lüscher and P. Weisz, JHEP 0109 (2001) 010.

[18] F. Gliozzi, M. Pepe, and U.-J. Wiese, Phys. Rev. Lett. 104 (2010) 232001.

[19] A. Athenodorou, B. Bringoltz, and M. Teper, Phys. Lett. B656 (2007) 132.

[20] P. Bialas, L. Daniel, A. Morel, and B. Petersson, Nucl. Phys. B836 (2010) 91.

[21] A. Allais and M. Caselle, JHEP 0901 (2009) 073.

[22] M. Caselle, arXiv:1004.3875 [hep-lat].

[23] A. S. Bakry, D. B. Leinweber, P. J. Moran, A. Sternbeck, and A. G. Williams, arXiv:1004.0782 [hep-lat].

[24] F. Gliozzi, M. Pepe, and U.-J. Wiese, arXiv:1006.2252 [hep-lat].

[25] M. Lüscher and P. Weisz, JHEP 0407 (2004) 014. 
[26] O. Aharony and E. Karzbrun, JHEP 0906 (2009) 012.

[27] O. Aharony and M. Field, arXiv:1008.2636 [hep-th].

[28] O. Aharony and N. Klinghoffer, arXiv:1008.2648 [hep-th].

[29] K. Dietz and T. Filk, Phys. Rev. D27 (1983) 2944.

[30] R. Sommer, Nucl. Phys. B411 (1994) 839. 